Check for updates

Cite this: RSC Adv., 2017, 7, 38915

\title{
Tailoring silica-rubber interactions by interface modifiers with multiple functional groups
}

\author{
Zheng Sun, ${ }^{a}$ Qiang Huang, ${ }^{c}$ Liqun Zhang, (D) ab Youzhi Wang ${ }^{\mathrm{C}}$ and Youping Wu (D) *ab
}

The filler-rubber interface is crucial in preparing ideal filled rubber composites. Interface modifiers are typically used for enhancing silica-rubber interactions and facilitating silica dispersion. In this work, trimethylolpropane tris(3-mercaptopropionate) (TMPMP) was found to be particularly effective in dispersing silica and the mechanism was investigated. Furthermore, the mercapto groups of TMPMP were reacted in gradient with the vinyl groups of vinyltriethoxysilane (VTES), which formed a series of interface modifiers with enhanced modifier-silica interactions and reduced modifier-rubber interactions. After applying the modifiers in silica filled rubber composites, the Payne effect, bound rubber content, dispersion morphology, mechanical and viscoelastic properties were studied. The results indicated that the interface modifiers with multiple functional groups were capable of purposefully balancing the interactions between rubber and silica, which brought about flexibility to optimize composite properties.

Received 3rd July 2017

Accepted 3rd August 2017

DOI: 10.1039/c7ra07321f

rsc.li/rsc-advances

the modification. However, the volatile organic by-products and the high energy consumption during the in situ reaction should be reduced. It is also striking that some other modifiers form hydrogen bonds with silica. ${ }^{10-13}$ Although the hydrogen bonds are relative weak and unstable, the feature of spontaneous formation is favorable. Besides typical oxygenous groups, imidazolium ionic liquids were substantiated outstanding for silica dispersion in mechanism of hydrogen bonding. ${ }^{13,14}$ A comparison was carried out by esterification or simply mixing carboxyl modifier with silica, and the results suggest that the unstable hydrogen bonding led to lower tensile modulus but higher tear strength than covalent bonding, indicating more slippage of rubber on the surface of silica. ${ }^{15}$ Moreover, a combined experiment and molecular dynamics simulation was used to study the effect of carboxyl modification of rubber on silica-rubber interactions. ${ }^{16,17}$ The simulation showed an optimum value of modifier content and a maximal silanol-carboxyl hydrogen bonds number, which implied that the carboxyl-carboxyl hydrogen bonds may compete with the silanol-carboxyl hydrogen bonds.

For modifier-rubber interactions, the sulfurated sliane such as bis-(triethoxysilylpropyl)-tetrasulfide and 3-benzothia-zolthio1-propyltriethoxylsilane can react with unsaturated rubbers during vulcanizing. ${ }^{18,19}$ Moreover, mercapto-containing modifiers such as 3-mercaptopropyl-ethoxy-bis(tridecyl-pentaethoxysiloxane) can react with rubber during mixing. ${ }^{20}$ The early formed covalent filler-rubber interactions are believed capable to draw back the silica from agglomerates under shear.

To coordinate modifier-silica and modifier-rubber interac-

${ }^{a}$ State Key Laboratory of Organic-Inorganic Composites, Beijing University of Chemical Technology, Beijing 100029, China. E-mail: wuyp@mail.buct.edu.cn; Fax: +86-1064456158; Tel: $+86-10-64442621$

tions, two modifiers with an additional siloxy group or an additional capped mercapto group were compared with 3-octanoylthio-1-propyltriethoxysilane. ${ }^{21}$ This experiment was supposed to evaluate the synergistic and balanced effect of 
multiple functional groups in one modifier, but the complex latent variable made it hard to analysis.

To our knowledge, few researches focused on tailoring and modulating the modifier-rubber and modifier-silica interactions by comparing series of gradually varied modifier structures. The present work took advantages of interface modifiers with multiple functional groups to find the appropriate strength and the strength allocation of the interactions. Concretely, trimethylolpropane tris(3-mercaptopropionate) (TMPMP) containing triple mercapto and triple ester groups was investigated as an interface modifier. The modifier-rubber interactions and the modifier-silica interactions were carried out by thiol-ene click chemistry and ester-silanol hydrogen bonds, respectively. The benefit of triple functional groups was studied by comparing TMPMP with two single functional modifiers. Furthermore, the single, double and triple vinyltriethoxysilane (VTES) modified TMPMP were synthesized by thiol-ene click reaction, which enhanced the modifier-silica interactions and reduced the modifier-rubber interactions in gradient, aiming to find the sweet spot of the interactions and tailor the properties of composites.

\section{Experimental}

\section{Materials}

Commercial silicone rubber (SiR) 110-9S with vinyl content of $5 \mathrm{~mol} \%$ was purchased from Ningbo Daoruo Silicone, China. Commercial solution polymerized styrene-butadiene rubber (SSBR) 2557TH (styrene content of $25 \mathrm{wt} \%$ and vinyl content of $43 \mathrm{wt} \%$, extended with $37.5 \mathrm{wt} \%$ of oil) was purchased from Dushanzi Petrochemical Sinopec, China. TMPMP was the product of Sigma-Aldrich. Ethyl mercaptoacetate (EMA) and 1,2-ethanedithiol (EDT) were the products of Aladdin Industrial Corporation. Precipitated silica ULTRASIL VN3 was purchased from Evonik Industries AG. VTES was the product of Energy Chemical, China. All the other ingredients, such as 2,5-bis(tert-butylperoxy)2,5-dimethylhexane (DBPMH), stearic acid (SA), $N$-isopropyl- $N$ phenyl-p-phenylenediamine (4010NA), poly(1,2-dihydro-2,2,4trimethylquinoline) (RD) and tetrahydrofuran (THF) were all commercially available industrial products. The molecular structures of TMPMP, EDT, EMA, and VTES are shown in Fig. 1.

\section{Preparation and characterization of silica/TMPMP and silica/ EMA mixtures}

To investigate the modifier-silica interactions, the mixtures of silica/TMPMP and silica/EMA were prepared. The THF, silica

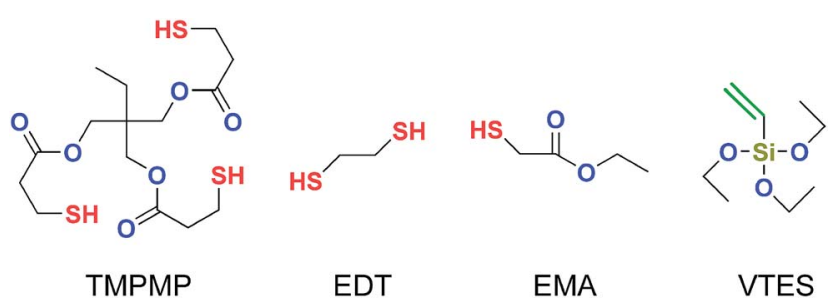

Fig. 1 Molecular structures of TMPMP, EDT, EMA and VTES. and TMPMP or EMA were mixed at weight ratio of $100: 10: 1$ and kept stirring for 2 hours at room temperature. Then the suspensions were centrifuged to get the precipitates. The precipitates were then Soxhlet extracted by THF for 24 hours to wash out the non-covalent absorbed TMPMP or EMA. The precipitates before and after extraction were dried respectively for taking the FTIR (Tensor 27 spectrometer, Bruker Optik Gmbh Co., Germany) spectra measurement. The samples were grinded with $\mathrm{KBr}$ and pressed into tablet for transmitted measurement with wavenumber ranged in $400-4000 \mathrm{~cm}^{-1}$.

\section{Preparation of silica/SSBR compounds with TMPMP, EDT and EMA}

To investigate the mechanism of TMPMP on dispersing silica and silica-rubber interactions, silica filled SSBR compounds with TMPMP, EDT and EMA were prepared. 137.5 parts per hundred parts of rubbers (phr) of SSBR, 50 phr of silica, 1 phr of SA and 0.5 phr of TMPMP or 0.178 phr of EDT or $0.452 \mathrm{phr}$ of EMA (equivalent in moles of mercapto groups) were mixed respectively on a two-roll mill at room temperature. A blank reference without modifier was also prepared. Then each of four compounds was sheared and heated at $100{ }^{\circ} \mathrm{C}$ for $8 \mathrm{~min}$ on another two-roll mill to assure the thiol-ene click reaction. After cooling to room temperature, $1.5 \mathrm{phr}$ of RD, $1.5 \mathrm{phr}$ of $4010 \mathrm{NA}$ and 0.5 phr of DBPMH were mixed well with each of four compounds.

\section{Preparation and characterization of VTES modified TMPMP}

First, TMPMP and VTES were dissolved in THF as $50 \mathrm{wt} \%$ solutions respectively. Then the solutions were mixed together at room temperature with stirring, and the mole ratios of TMPMP and VTES were controlled at $1: 1,1: 2$ and $1: 3$, respectively. After the mixing, the mixtures were further reacted at $60{ }^{\circ} \mathrm{C}$ for 6 hours with stirring. The products of this series of VTES modified TMPMP were denominated as $\mathrm{TV}_{1}, \mathrm{TV}_{2}$ and $\mathrm{TV}_{3}$, respectively. The schematic of the reactions is shown in Fig. 2.

The products were dissolved in $\mathrm{CDCl}_{3}$ and characterized by ${ }^{1} \mathrm{H}-\mathrm{NMR}$ spectrometer (Bruker Avance III Digital NMR spectrometer, $400 \mathrm{MHz}$, Bruker, Germany).

\section{Preparation of composites containing interface modifiers with multiple functional groups}

The procedures of preparation were as follows:

(1) Masterbatches of SiR, silica and hydroxyl silicone oil at mass ratio of $100: 50: 1$ were mixed on two-roll mill at room temperature.

(2) Masterbatches containing $20 \mathrm{phr}$ of SiR were mixed with $110 \mathrm{phr}$ of SSBR, $50 \mathrm{phr}$ of silica, $1 \mathrm{phr}$ of SA on two-roll mill at room temperature. The compound added with $0.5 \mathrm{phr}$ of TMPMP was identified as $\mathrm{TV}_{0}$, and the reference compound was identified as $T_{0} V$. The synthesized products of $T_{1}, T V_{2}$ and $T V_{3}$ were respectively added to compounds in equivalent moles to TMPMP.

(3) The compounds were heat-treated respectively on another two-roll mill at $100{ }^{\circ} \mathrm{C}$ for $8 \mathrm{~min}$. 


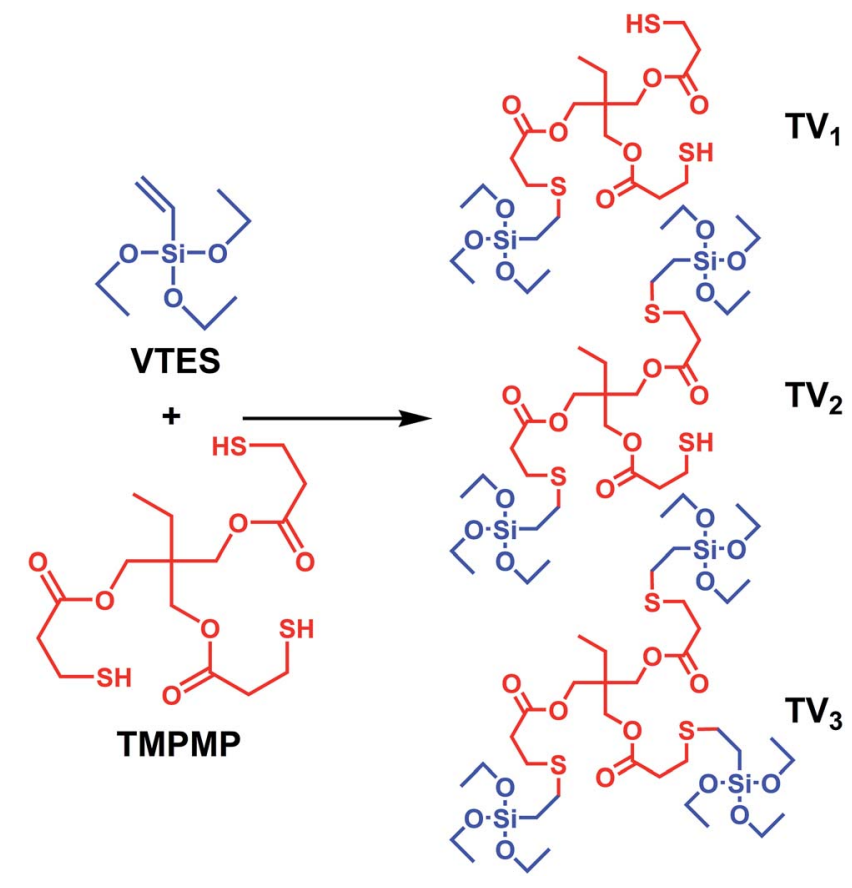

Fig. 2 Reaction formulas of synthesizing $T V_{1}, T V_{2}$ and $T V_{3}$.

(4) Complement VTES to 5.6 phr (eliminating the difference among the VTES modified to TMPMP) and silica to $70 \mathrm{phr}$ at room temperature on two-roll mill.

(5) The compounds were heat-treated respectively on two-roll mill at $145{ }^{\circ} \mathrm{C}$ for $8 \mathrm{~min}$.

(6) After cooling to room temperature, $1.5 \mathrm{phr}$ of $\mathrm{RD}, 1.5 \mathrm{phr}$ of $4010 \mathrm{NA}$ and $0.5 \mathrm{phr}$ of DBPMH were mixed well with each of five compounds.

(7) The compounds were vulcanized at $170{ }^{\circ} \mathrm{C}$ for $20 \mathrm{~min}$.

\section{Characterization and measurement of the composites}

The compounds were soaked in THF to determine the bound rubber content. The total soaking time was 5 days and the solvent was changed at the third day. The insoluble gel was dried in oven at $60{ }^{\circ} \mathrm{C}$. Then the thermal weigh loss of the dried silica with bound rubber was measured. The measurement was taken by TGA (Mettler-Toledo Co., Switzerland) under nitrogen atmosphere with temperature range of $30-700{ }^{\circ} \mathrm{C}$ and heating rate of $10{ }^{\circ} \mathrm{C} \mathrm{min}^{-1}$. The bound rubber content was finally converted in unit of phr.

The dynamic storage moduli of the compounds were measured by a Rubber Process Analyzer (RPA2000, Alpha Technological, USA). Strain sweep from 0.28 to $400 \%$ was carried out at $100{ }^{\circ} \mathrm{C}, 1 \mathrm{~Hz}$.

The vulcanization characteristics were measured by an MRC3 rotorless rheometer (Beijing Ruidayuchen instrument, China) at $170{ }^{\circ} \mathrm{C}$.

The TEM microstructure morphology was observed via a Tecnai G220 (FEI Co., USA) with an accelerating voltage of $200 \mathrm{kV}$. The samples were cut by an ultra-microtome under liquid nitrogen quenching.
The tensile properties were measured on an electronic tensile machine (Shenzhen SANS Test Machine, China), according to ISO 37: 2011. The shore A hardness was measured according to ISO 868: 2003.

The dynamic mechanical properties were measured by a VA 3000 dynamic mechanical analyzer (DMA) (Metravib, France). The specimens were tested at temperature range from $-60^{\circ} \mathrm{C}$ to $80{ }^{\circ} \mathrm{C}$ with heating rate of $3{ }^{\circ} \mathrm{C} \mathrm{min}^{-1}$, and the tensile strain amplitude was $0.1 \%$ with frequency of $10 \mathrm{~Hz}$.

\section{Results and discussion}

\section{Mechanism of TMPMP improving silica-rubber interactions}

The TMPMP molecule was constituted by triple mercapto groups and triple ester groups. On one hand, the mercapto groups could efficiently react with unsaturated rubber through thiol-ene click reaction. ${ }^{\mathbf{1 3}, \mathbf{1 6}, \mathbf{1 7 , 2 0}}$ Besides establishing modifierrubber interactions, the triple mercapto groups could achieve a chain extension effect or even crosslink effect when reacted with different rubber chains. On the other hand, the triple ester groups could possibly form hydrogen bonds with silanol groups on the surface of silica, so that the modifier-silica interactions were expected as well. To separate the complex effects, EMA and EDT were used as reference molecules. The EMA had a single mercapto group and a single ester group, which could form modifier-silica and modifier-rubber interactions without chain extension effect. On the contrary, the EDT only had double mercapto groups that could extend the chains. A blank reference without modifier was also taken into comparison.

To investigate the silanol-ester interactions, the FTIR spectra of TMPMP or EMA treated silica were measured. The spectra are shown in Fig. 3. The treated silica before wash showed significant peaks at $1728 \mathrm{~cm}^{-1}$ and $1712 \mathrm{~cm}^{-1}$, which attributed to the $-\mathrm{C}=\mathrm{O}$ stretching vibrations in TMPMP and EMA respectively. The peaks shifted to lower wavenumber compared with the original ones at $1737 \mathrm{~cm}^{-1}$, which confirmed the existence of hydrogen bonds between silanol groups and ester groups. $^{22}$ The shift of $-\mathrm{C}=\mathrm{O}$ peak in TMPMP was smaller than that in EMA. This suggested the weaker hydrogen bond of each ester group on average, which probably attributed to the

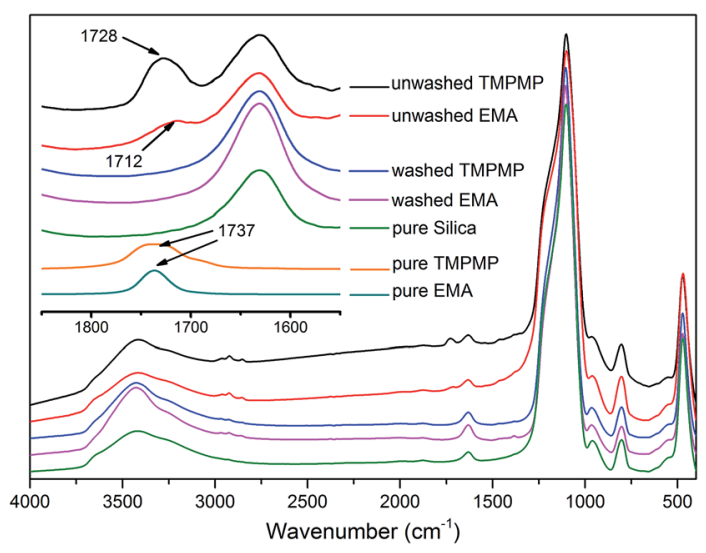

Fig. 3 FTIR spectra of TMPMP and EMA treated silica. 
steric hindrance. As the schematic diagram showed in Fig. 4, the triple ester groups could hardly form hydrogen bonds simultaneously. However, the strength of $-\mathrm{C}=\mathrm{O}$ peak in TMPMP was stronger. This suggested that the TMPMP had higher chance to be adsorbed due to the triple probability to form hydrogen bonds with silica. After the treated silica was washed by $\mathrm{THF}$, the peak of $-\mathrm{C}=\mathrm{O}$ disappeared and the spectra became consist with pure silica. It confirmed that the adsorption was attributed to hydrogen bonds rather than covalent bonds, and the ester-silanol hydrogen bonds were relative weak that could be disrupted by polar solvent. ${ }^{23}$

Fig. 5 shows the bound rubber content of silica/SSBR compounds. The compound with EMA had slight more bound rubber contents than the blank, which indicated that the weak modifier-silica interactions existed but probably was reduced by the solvation effect. The compound with TMPMP had evidently more bound rubber, whereas the EDT exhibited less than the blank. It confirmed that the bound rubber of TMPMP was attributed to silica-rubber interactions. The mechanism of TMPMP enhancing silica-rubber interactions was proposed: the high chances of the triple mercapto groups and triple ester groups to form covalent bonds with rubber and hydrogen bonds with silica, respectively. Considering that the amount of the three kinds of modifiers were equal in moles of mercapto groups, the high efficiency of the interface modifier with multiple functional groups was featured.

The storage modulus under small strain is associated with the filler-filler interactions and filler dispersion, which is denominated in Payne effect. ${ }^{24,25}$ The storage moduli of four compounds with the storage time of 14 and 21 days are shown in Fig. 6. It was significant that the compound with TMPMP had the lowest Payne effect at both the storage time of 14 and 21 days. Meanwhile, the modulus rising during storage was the lowest. This phenomenon suggested that only the triple ester groups in TMPMP had the chance to effectively break up the agglomerates of silica and the triple mercapto groups connected enough rubber chains to prevent the silica from re-agglomerating. The other three compounds exhibited similar Payne effect at storage time of 14 days, but the modulus rising of the compound with EMA was higher and the modulus rising of the compound with EDT was lower, suggesting that the EMA may break up the agglomerates at first, but hardly kept the silica dispersed during storage; the EDT

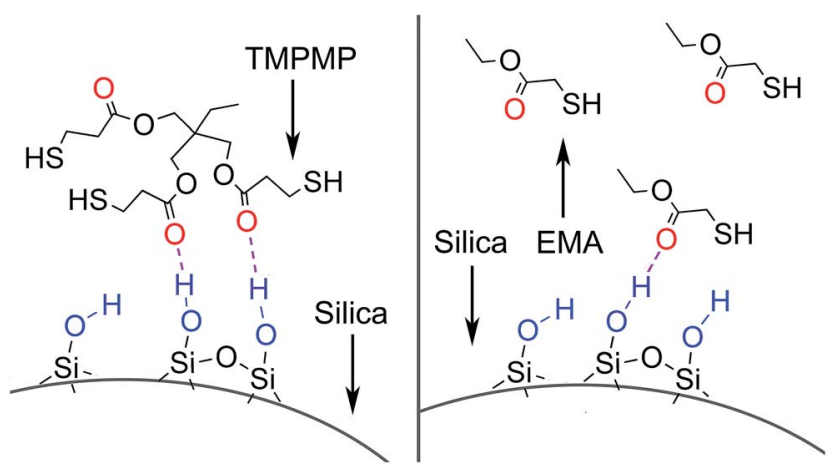

Fig. 4 Hydrogen bonds of TMPMP-silica and EMA-silica.

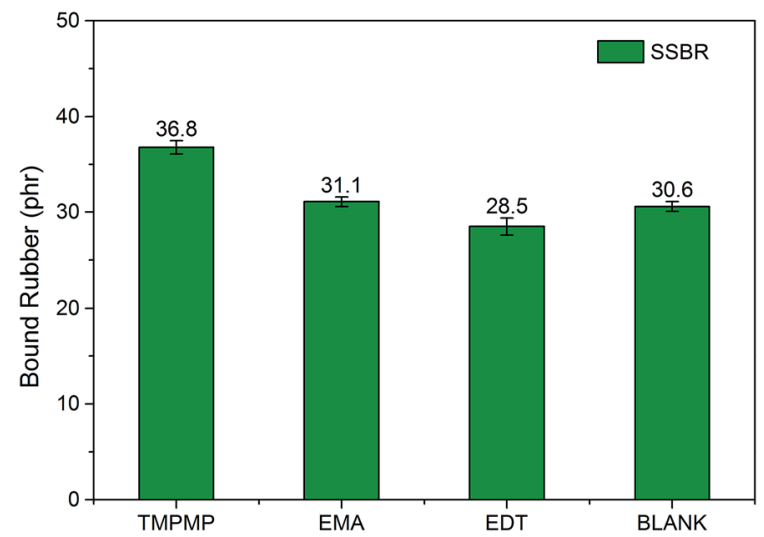

Fig. 5 Bound rubber content of the silica/SSBR compounds with TMPMP, EMA, EDT and blank.

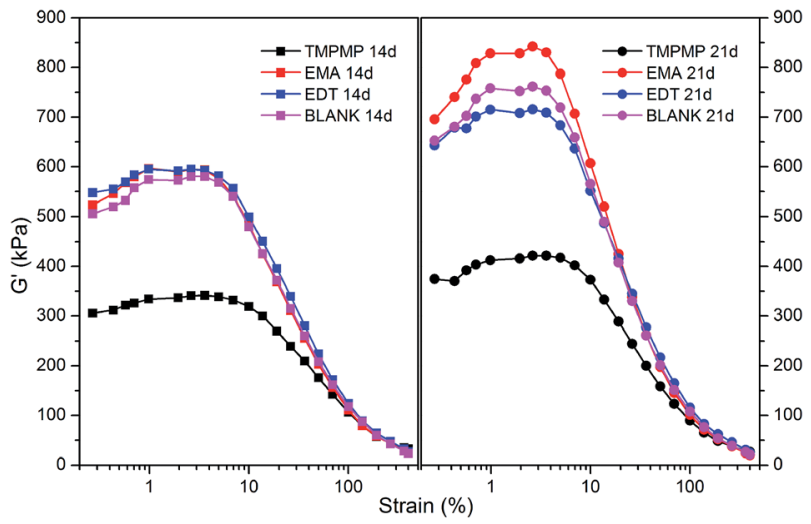

Fig. 6 Storage moduli of silica/SSBR compounds with TMPMP, EMA, EDT and blank.

hardly broke up the agglomerates but isolated the silica from further agglomerating. The low modulus rising at strain of 0.1$1 \%$ and broad modulus plateau of the compound with TMPMP also confirmed the strong silica-rubber interactions. ${ }^{17}$ These entire phenomena indicated that both the silica-rubber interactions and the chain extension effect were indispensable for silica dispersion and isolation. The benefit of interface modifier with multiple functional groups was highlighted again.

The filler dispersion morphology could validate the Payne effect analysis from microscopic view. Fig. 7 shows the microstructure morphology of the silica/SSBR composites. The composites with TMPMP (a) exhibited the best silica dispersion. The agglomerates were effectively broken. The composites with EMA (b) exhibited some large agglomerates, indicating that the EMA with single mercapto group and single ester group was inferior to the TMPMP with triple functional groups. The dispersion with EDT (c) was similar to the blank (d), showed the chain extension effect itself was futile in breaking up agglomerates.

\section{Synthesis and characterization of VTES modified TMPMP}

According to the results above, by adding TMPMP at $1 \%$ mass fraction of silica, the Payne effect decreased about $40 \%$, and 

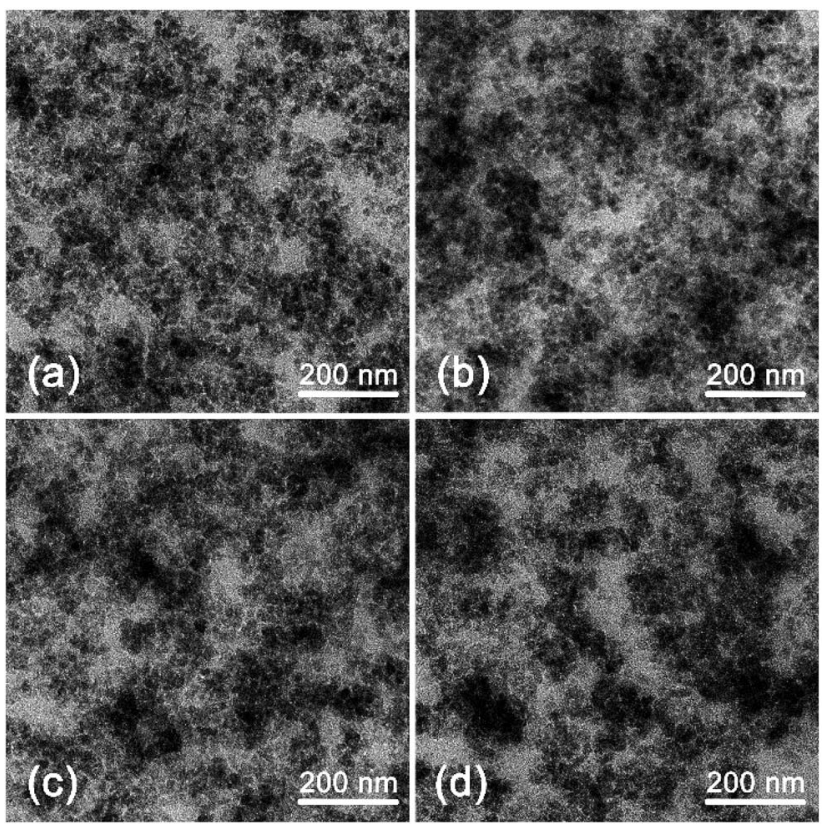

Fig. 7 Microstructure morphology of composites with TMPMP (a) EMA (b), EDT (c) and blank (d).

TMPMP couldn't be replaced by EMA or EDT. So TMPMP was a particularly high-efficiency modifier for improving silicarubber interactions and silica dispersion. Further considered the weak hydrogen bonds, VTES was introduced. The vinyl groups in VTES could react with mercapto groups in TMPMP, and the siloxy groups could form covalent bonds with silica to enhance the modifier-silica interactions. A series of interface modifiers were synthesized by reacting TMPMP and VTES in different mole ratio, aiming to optimize the modifier-silica and modifierrubber interactions. The schematic diagram is shown in Fig. 8.

The thiol-ene click reaction has the qualities of high yield, high selectivity and mild react condition. These qualities made the click reaction convenient for molecular design and func-

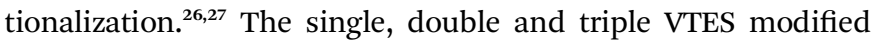
TMPMP were synthesized as reaction formulas showed in Fig. 2. The ${ }^{1} \mathrm{H}$-NMR spectra are shown in Fig. 9. All the $Y$-axes with VTES were normalized by height of the peaks at $1.23 \mathrm{ppm}$. The peak at $1.23 \mathrm{ppm}$ was the constant peak of $-\mathrm{CH}_{3}(\mathrm{C})$, which was chosen as reference to calculate the conversion of the vinyl groups. The elimination of $-\mathrm{HC}=\mathrm{CH}_{2}$ (A) signal at 5.8-6.2 ppm confirmed the reaction between vinyl groups and mercapto groups. The ratios of integral at 5.8-6.2 ppm to $1.23 \mathrm{ppm}$ were $0: 1,1: 92.1$ and $1: 14.1$ for $\mathrm{TV}_{1}, \mathrm{TV}_{2}$ and $\mathrm{TV}_{3}$, respectively. So that the conversions of vinyl groups were $100 \%, 97 \%$ and $79 \%$, respectively. The first two mercapto groups were easily reacted with VTES, which was benefited by the high yield of thiol-ene click reaction. But the last one was relative low-reactive owing to the steric hindrance.

\section{Application of VTES modified TMPMP in silica filled rubber composites}

Fig. 10 shows the bound rubber contents of the compounds with the series of VTES Modified TMPMP. With the assist of

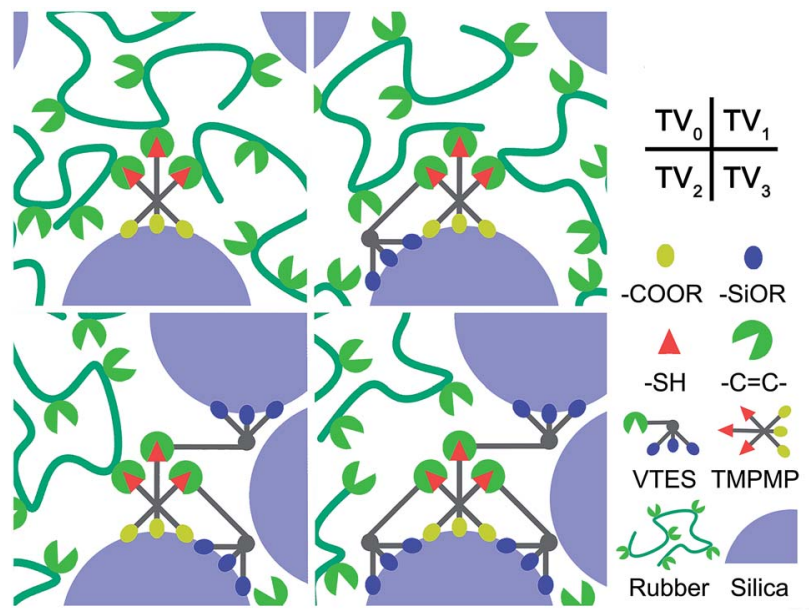

Fig. 8 Schematic diagram of silica-rubber interactions with VTES modified TMPMP.

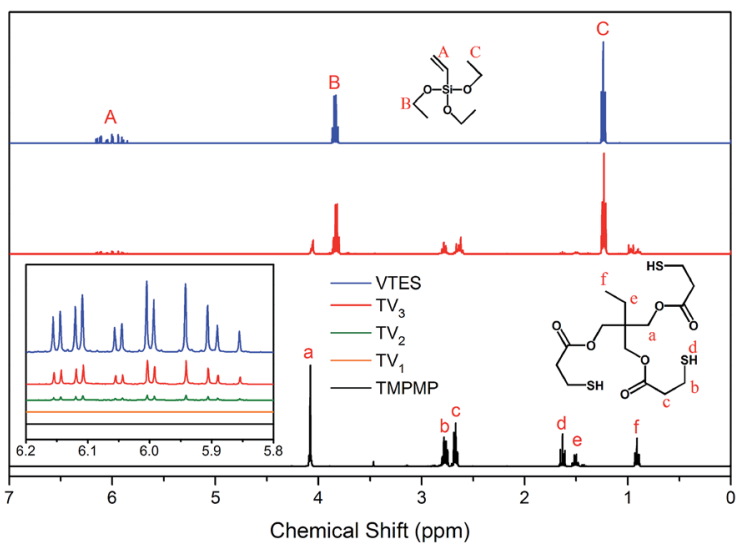

Fig. $9{ }^{1} \mathrm{H}$-NMR spectra of VTES modified TMPMP.

TGA, the SSBR and SiR could be distinguished by the different decomposition temperature. The ratio of the two rubbers in bound rubber could reflect the phase distribution of silica. ${ }^{28,29}$ Due to the high heat resistance of SiR, the weight loss steps of the two rubbers weren't overlapped. As shown in Fig. 11, the step of $290{ }^{\circ} \mathrm{C}$ to $490{ }^{\circ} \mathrm{C}$ was attributed to weight loss of SSBR and the step of $490{ }^{\circ} \mathrm{C}$ to $650{ }^{\circ} \mathrm{C}$ was attributed to SiR. According to Fig. 10, the amounts of $\mathrm{SiR}$ bound rubber were almost independent of the modifier structures. The rare concentration of vinyl groups in SiR limited the reactivity with mercapto groups in modifiers. However, the amounts of SSBR bound rubber were depended on the structure of the interface modifiers evidently. Compared to the reference with only VTES added, the additional TMPMP led to larger bound rubber content, in accordance with the result in the first section. When one of the mercapto groups in TMPMP was modified by VTES, the ability to couple the rubber chains was supposed to decrease. However, the bound rubber increased due to the covalent modifier-silica interactions, which couldn't be disrupted by solvent. As more mercapto groups were modified, the bound rubber decreased, indicating the loss on modifier- 


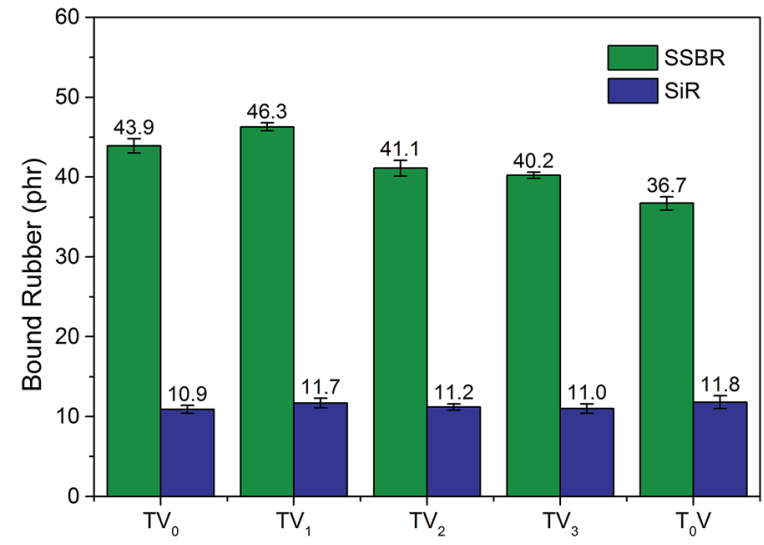

Fig. 10 Bound rubber contents of the compounds with VTES modified TMPMP.

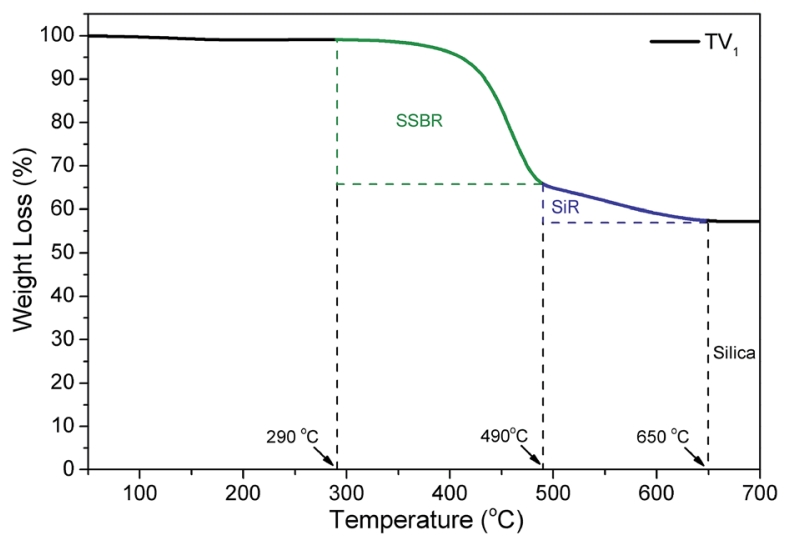

Fig. 11 Thermo weight loss of silica/bound rubber gel with modifier of $\mathrm{TV}_{1}$.

rubber interactions outweighed the gain on modifier-silica interactions.

The storage moduli of the compounds with VTES modified TMPMP are shown in Fig. 12. The trend was clear that the Payne effect became stronger as the more of mercapto groups in TMPMP modified by VTES. It was mostly agreed to the trend of bound rubber content with the only exception that the Payne effect with TMPMP was weaker than that with $\mathrm{TV}_{1}$. This phenomenon could be accounted for the properties of hydrogen bonds. The hydrogen bonds possibly were enough to break up the agglomerates during mixing to reduce the Payne effect, whereas those were disrupted by solvent so that the bound rubber was less. In respect of Payne effect, the stronger modifier-rubber interactions led to the better silica dispersion and isolation, so that the reduced filler-filler interactions and Payne effect. The breadth of modulus plateau also reflected that the silica-rubber interactions were dominated by modifier-rubber interactions in presence of the triple hydrogen bonds.

Fig. 13 shows the microstructure morphology of composites with the series of VTES modified TMPMP. The composites with TMPMP (a) and $\mathrm{TV}_{1}$ (b) exhibited better silica dispersion and fewer agglomerates. On the contrary, the reference one with

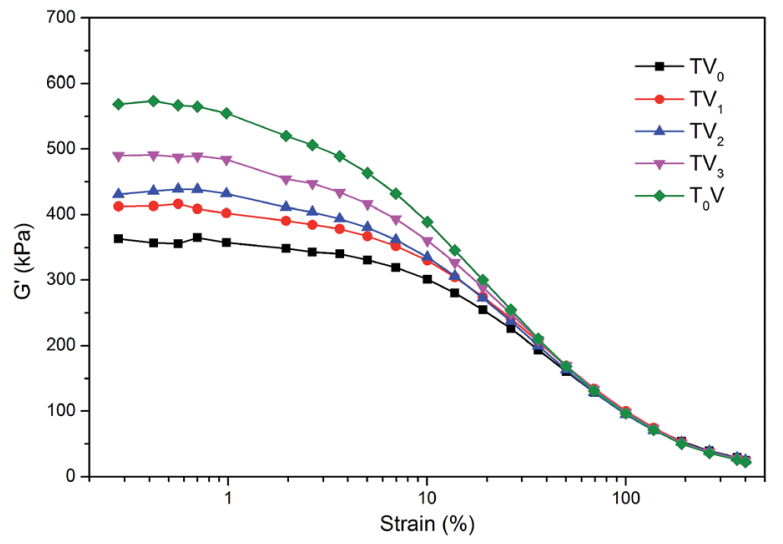

Fig. 12 Storage moduli of compounds with VTES modified TMPMP.

only VTES (e) exhibited a mass of large agglomerates. The dispersion with $\mathrm{TV}_{2}$ (c) and $\mathrm{TV}_{3}$ (d) was in the middle. All the dispersion morphologies were corresponding to the previous Payne effect analysis. The morphology of the composites showed the tendency that the more rubber chains involved by interface modifiers, the better dispersion exhibited.

The curing curves of the compounds with the series of VTES modified TMPMP are shown in Fig. 14. The torque rising trends among the compounds were similar, indicating that the mercapto groups wouldn't impact curing because they were fully reacted during heat treatment. The consistent crosslink density also set a convincing baseline to study the influence of silica dispersion and silica-rubber interactions. Although the TMPMP and $\mathrm{TV}_{1}$ could extend the rubber chains, the minimum torque were lower than other compounds. The result was attributed to the correlation between the minimum torque and the storage modulus at low strain. The dispersion of silica was predominant in this situation.

The static mechanical properties are shown in Table 1. Since the overall amounts of interface modifiers in compounds (except $T_{0} V$ ) were exactly the same, the mechanical properties varied in a small range. The only difference between the compounds was the targets that the mercapto groups coupled with. Although the dispersion of silica was the best with TMPMP (according to Payne effect and morphology), the tensile strength and stress under larger deformation were lower than the compound with $\mathrm{TV}_{1}$. It was owing to the instability of the hydrogen bonds, which gave the rubber chains chance to slide on the surface of silica. ${ }^{14}$ When TMPMP was modified by more than one VTES, the moduli dropped again because of the decreased interactions with rubber. ${ }^{30}$ Even the coupling with different silica to form agglomerates was possible for them, which reduced the effective amount of silica. The reference without TMPMP showed the lowest modulus indicating that the vinyl groups of VTES could hardly form covalent bonds under the relatively low crosslink density. In general, the single VTES modified TMPMP exhibited the best mechanical properties of vulcanizate, suggested that the balanced silica-rubber covalent interactions were necessary. The limitation of hydrogen bonds was the instability under large deformation. 

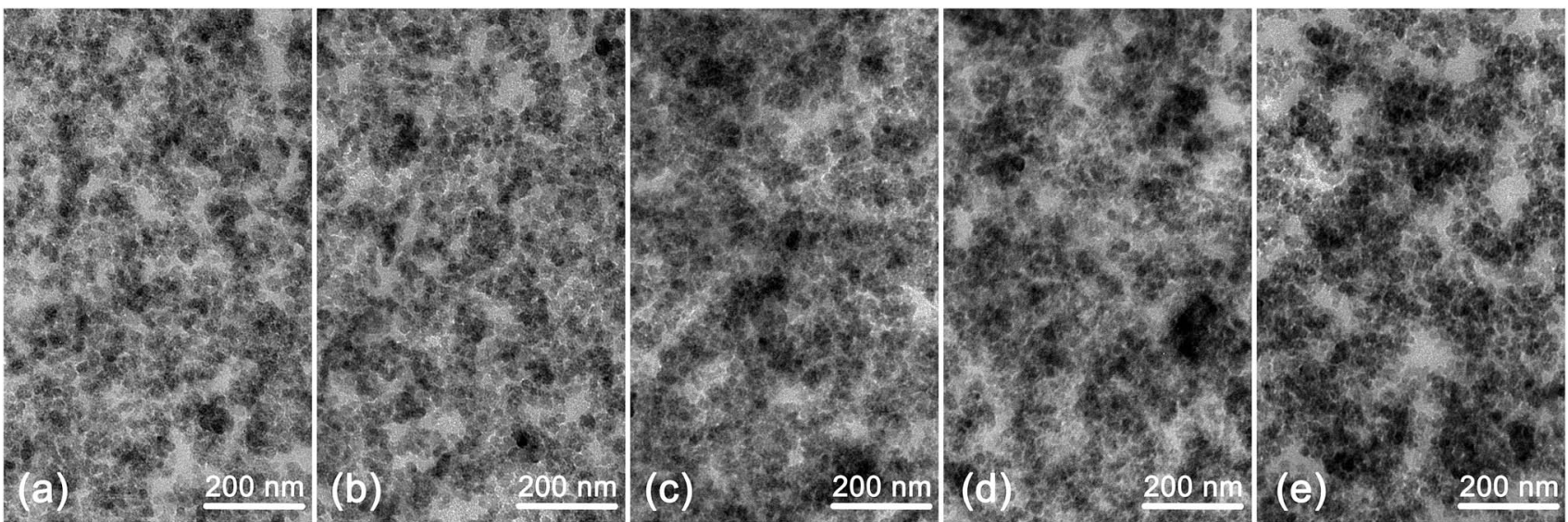

Fig. 13 Microstructure morphology of composites of $\mathrm{TV}_{0}(\mathrm{a}), \mathrm{TV}_{1}(\mathrm{~b}), \mathrm{TV}_{2}$ (c), $T \mathrm{~V}_{3}(\mathrm{~d})$, and $\mathrm{T} 0 \mathrm{~V}(\mathrm{e})$.

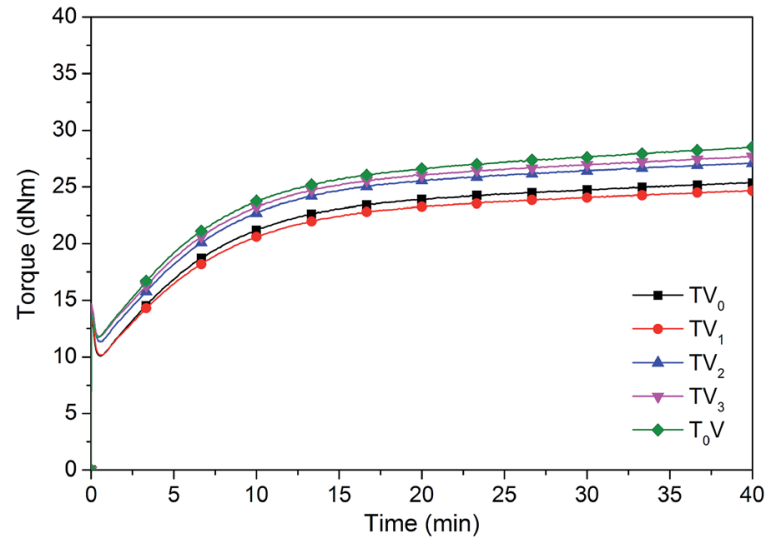

Fig. 14 Curing curves of compounds with VTES modified TMPMP

The viscoelasticity of the filled rubber composites was not only determined by the structure of rubber macromolecule but also the dispersion of filler and the filler-rubber interface. The loss factor $\tan \delta$ versus temperature curves are shown in Fig. 15. As expected, the composites with TMPMP and $\mathrm{TV}_{1}$ exhibited the high $\tan \delta$ at glass transition zone and low $\tan \delta$ at high temperature, indicating the better filler dispersion and stronger filler-rubber interactions. ${ }^{17}$ These properties are eagerly needed by tire industry which mean higher wet-skid resistance and lower rolling resistance. The measurement was taken under strain of $0.3 \%$ so that the instability of hydrogen bonds was non-significant. The compound with $\mathrm{TV}_{0}$ exhibited the most ideal dynamic mechanical properties.

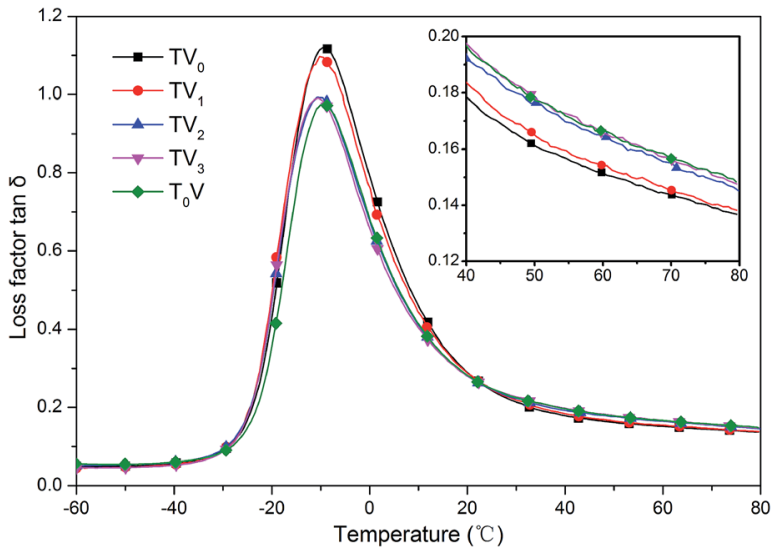

Fig. 15 Loss factor $\tan \delta$ vs. temperature curves of composites with VTES modified TMPMP.

Analyzing the results above, the dynamic mechanical properties under small deformation and Payne effect were consistent to the dispersion morphology, whereas the tensile moduli under high strain were strongly associated to the bound rubber contents. It was because that the properties under small deformation were mostly controlled by filler-filler interactions ${ }^{24}$ where the modifier played the role of breaking up agglomerates and isolating them from re-agglomerating, so that the relative weak modifier-silica interactions were compromised in order to ensure the triple mercapto groups coupling more rubber chains. On the contrary, the mechanical properties under high strain were mostly controlled by filler-rubber

Table 1 Static mechanical properties of composites with VTES modified TMPMP

\begin{tabular}{|c|c|c|c|c|c|}
\hline Samples & $\mathrm{TV}_{0}$ & $\mathrm{TV}_{1}$ & $\mathrm{TV}_{2}$ & $\mathrm{TV}_{3}$ & $\mathrm{~T}_{0} \mathrm{~V}$ \\
\hline Tensile strength (MPa) & $12.5 \pm 0.3$ & $12.8 \pm 0.3$ & $12.6 \pm 0.3$ & $12.2 \pm 0.3$ & $12.6 \pm 0.2$ \\
\hline Elongation at break (\%) & $436 \pm 26$ & $418 \pm 32$ & $443 \pm 25$ & $457 \pm 21$ & $517 \pm 15$ \\
\hline Modulus at $100 \%(\mathrm{MPa})$ & $1.8 \pm 0.1$ & $1.8 \pm 0.1$ & $1.8 \pm 0.1$ & $1.7 \pm 0.1$ & $1.6 \pm 0.1$ \\
\hline Modulus at $300 \%(\mathrm{MPa})$ & $7.8 \pm 0.2$ & $8.4 \pm 0.1$ & $7.9 \pm 0.2$ & $7.4 \pm 0.1$ & $6.5 \pm 0.2$ \\
\hline Shore A hardness & 59 & 59 & 61 & 61 & 60 \\
\hline
\end{tabular}


interactions, so that the covalent bonding was necessary to limit the slippage of rubber chains on the surface of silica. ${ }^{30}$ As the diversified properties required different structures of interface modifiers, the adaptability of the modifiers with multiple functional groups were highly desired.

\section{Conclusions}

The mechanism of TMPMP enhancing silica-rubber interactions were proved to be the ester-silanol hydrogen bonds improving modifier-silica interactions and the thiol-ene click reaction improving modifier-rubber interactions. By comparing TMPMP with EMA and EDT, the triple ester groups increased the chance of hydrogen bonding for each of TMPMP molecules, and the triple mercapto groups coupled more rubber chains to isolate the silica from re-agglomerate, so that the synergistic benefit of multiple functional groups were proposed. Furthermore, the VTES modified TMPMP were synthesized by thiol-ene click reaction to study the ideal balance between modifier-silica and modifier-rubber interactions. The triple ester groups were found effective for silica dispersion, and the enhancement of modifier-rubber interactions surpassed modifier-silica interactions when considering dynamic properties under small deformation. However, the appropriate covalent bonding between silica and rubber benefit the tensile strength and stress under larger deformation by reducing the slippage at the interface. In conclusion, the easily-synthesized, high-efficiency and custom-made interface modifiers could be tailored to fit different kinds of rubber and silica to achieve optimized composites properties.

\section{Acknowledgements}

The financial support of the National Key Research and Development Program of China (No. 2017YFB0307000) is gratefully acknowledged.

\section{References}

1 D. C. Edwards, J. Mater. Sci., 1990, 25, 4175-4185.

2 S. Wollf, M. J. Wang and E. H. Tan, Kautsch. Gummi Kunstst., 1994, 47, 105.

3 J. Fröhlich, W. Niedermeier and H.-D. Luginsland, Composites, Part A, 2005, 36, 449-460.

4 M. J. Wang, S. Wolff and E. H. Tan, Rubber Chem. Technol., 1993, 66, 178-195.

5 M. J. Wang, Rubber Chem. Technol., 1998, 71, 520-589.
6 J. H. Ma, S. H. Zhao, L. Q. Zhang and Y. P. Wu, Rubber Chem. Technol., 2013, 86, 664-678.

7 M. J. Wang, Rubber Chem. Technol., 2008, 81, 552-575.

8 M. J. Wang, S. Wolff and J. B. Donnet, Rubber Chem. Technol., 1991, 64, 559-576.

9 M. J. Wang and S. Wolff, Rubber Chem. Technol., 1992, 65, 715-735.

10 K. Katueangngan, T. Tulyapitak, A. Saetung, S. Soontaranon and N. Nithi-Uthai, Procedia Chem., 2016, 19, 447-454.

11 S. Mohapatra, R. Alex and G. B. Nando, J. Appl. Polym. Sci., 2016, 133, 43057.

12 P. Boochathum and N. Rongtongaram, J. Appl. Polym. Sci., 2016, 133, 43076.

13 Y. Lei, Z. Tang, L. Zhu, B. Guo and D. Jia, J. Appl. Polym. Sci., 2011, 123, 1252-1260.

14 J. Wang, H. Jia, L. Ding and X. Xiong, Polym. Adv. Technol., 2015, 26, 1168-1175.

15 M. C. W. Somaratne, N. M. V. K. Liyanage and S. Walpalage, J. Appl. Polym. Sci., 2014, 131, 40380.

16 Y. Luo, L. Qu, H. Su, T. W. Chan and S. Wu, RSC Adv., 2016, 6, 14643-14650.

17 L. Qu, L. Wang, X. Xie, G. Yu and S. Bu, RSC Adv., 2014, 4, 64354-64363.

18 L. Qu, G. Yu, L. Wang, C. Li, Q. Zhao and J. Li, J. Appl. Polym. Sci., 2012, 126, 116-126.

19 H. Peng, L. Liu, Y. Luo, H. Hong and D. Jia, J. Appl. Polym. Sci., 2009, 112, 1967-1973.

20 Y. C. Tao, B. Dong, L. Q. Zhang and Y. P. Wu, Rubber Chem. Technol., 2016, 89, 526-539.

21 J. Y. Ko, K. Prakashan and K. K. Jin, J. Elastomers Plast., 2012, 44, 549-562.

22 V. Bounor-Legaré, C. Angelloz, P. Blanc, P. Cassagnau and A. Michel, Polymer, 2004, 45, 1485-1493.

23 R. Wang, J. Ma, X. Zhou, Z. Wang, H. Kang, L. Zhang, K. Hua and J. Kulig, Macromolecules, 2012, 45, 6830-6839.

24 A. R. Payne, J. Appl. Polym. Sci., 1965, 9, 2273-2284.

25 R. Yang, Y. Song and Q. Zheng, Polymer, 2017, 116, 305-313.

26 C. E. Hoyle and C. N. Bowman, Angew. Chem., Int. Ed., 2010, 49, 1540-1573.

27 M. J. Kade, D. J. Burke and C. J. Hawker, J. Polym. Sci., Part A: Polym. Chem., 2010, 48, 743-750.

28 H. H. Le, S. Ilisch, D. Heidenreich, A. Wutzler and H. Radusch, Polym. Compos., 2010, 31, 1701-1711.

29 H. H. Le, S. Ilisch, G. R. Kasaliwal and H. J. Radusch, Rubber Chem. Technol., 2008, 81, 767-781.

30 J. W. T. Brinke, P. J. V. Swaaij, L. A. E. M. Reuvekamp and J. W. M. Noordermeer, Rubber Chem. Technol., 2003, 76, 12-35. 